\title{
Multi Spectral Image Classification Method with Selection of Independent Spectral Features through Correlation Analysis
}

\author{
Kohei Arai \\ Graduate School of Science and Engineering \\ Saga University \\ Saga City, Japan
}

\begin{abstract}
Multi spectral image classification method with selection processes of independent spectral features through correlation analysis is proposed. The proposed method is validated by applying to the polarimetric Synthetic Aperture Radar: SAR data. Also Probability Distribution Function: PDF for of features are checked and confirmed the most independent PDF allows greatest classification performance.
\end{abstract}

Keywords- image classification; polarimetric SAR; correlation analysis;

\section{INTRODUCTION}

Due to the fact that the Synthetic Aperture Radar image contains not so small noises, speckle noise in particular, classification is not so easy. There are so many methods for speckle noise removals. After the speckle noise is removed, it still difficult to get high classification performance.

From full polarimetric SAR images, not so small number of features can be derived in comparison to the single polarization of SAR images. It is possible to select independent feature among the not so small number of features. The classification method proposed here is to apply feature selection in accordance with the correlation among the features derived from full polarimetric SAR imagery data.

Radar polarimetry allows measurement the physical characteristics such as di-electric constant, slope of the ground cover targets as well as directionality of artificial objects by using scattering mechanism between electromagnetic (EM) wave and the targets [1], [2]. Polarimetric SAR image classification with the following three components of the polrimetric SAR data, (1) transmit Electro-magnetic wave with Horizontal Polarization(H-Pol) and receive the echo from the ground with $\mathrm{H}-\mathrm{Pol}(\mathrm{HH})$, (2) transmit Electro-magnetic wave with $\mathrm{H}-\mathrm{Pol}$ and receive the echo with Vertical Polarization(V-Pol)(HV) and (3) transmit Electro-magnetic wave with $\mathrm{V}$-Pol and receive the echo with $\mathrm{V}$-pol(VV) is widely available [3],[4]. On the other hand, the extraction of the scattering characteristics of the targets of interest by applying eigen value decomposition to the covariance matrix derived from the scattering matrix which is calculated from the three components are proposed [5]. Furthermore, the classification methods with the single / double /multiple, odd / even / diffuse, and odd / even / Bragg / multiple scattering components derived from the eigen value decomposition were proposed [6] while the classification methods with the sphere / deplane / helix, and sphere / Bragg / double of scattering components which are based on the spherical polarization which are derived from the scattering matrix were also proposed [7],[8].

Aforementioned proposed methods were reviewed [9]. Moreover, the classification method with the entropy $(\mathrm{H})$ which is defined with the sum of the first to third eigen values and the ratio of each eigen values, the anisotropy (A) which is defined as the ratio of sum and subtraction of the second and the third eigen values and $\operatorname{cosine} \alpha(\cos (\alpha))$ which is defined with the elements of the eigen vector corresponding to the first eigen value which is called coherency matrix( 3 by 3 ) was proposed by E.Pottier [10].

The application of these methods to sea ice discrimination (such as thin ice (TI), smooth first year ice (SF), rough first year ice $(\mathrm{RF})$ and open water $(\mathrm{OW})$ ) with the polarimetric SAR were attempted by using $\mathrm{H}, \mathrm{A}$, and $\cos (\alpha)$ [11]. Classification performance, however, were not satisfactory (20-40\% of classification errors were occurred for the classification of sea ice into four classes, ridged, compressed, new forming and smooth surface due to the fact that scattering mechanism based features were not used effectively. Meanwhile polarimentirc SAR image classification with polarization signature which are derived from Stokes or Muller or scattering matrix is widely available [12]. Polarization signature represents the scattering mechanism, in particular, surface roughness of the targets in concern.

One of the problems on the classification with polarization signature is classification performance. The method for effective utilization of polarization signature is still unclear to improve classification performance. The method proposed here is for extraction of effective information from the polarization signature by transforming the polarization signature onto an eigen space (eigen value decomposition). As the results from the eigen value decomposition which corresponds to the largest eigen value, a trajectory can be drawn. The trajectory represents the scattering mechanism in concern so that the largest curvature of the trajectory represents the most effective representatives of the scattering mechanism of the target of interest [13]. This is the theoretical background to propose the utilization of maximum curvature of the trajectory in an eigen space which is derived from the 
polarization signature to the sea ice classification.

The following section, the proposed method is described followed by the experiments. Then conclusion is described together with some discussions.

\section{PROPOSED METHOD}

\section{A. Principle of Polarimetric SAR}

Sending and receiving EM signals are expressed in Equation (1).

$$
\left(\begin{array}{c}
E_{h} \\
E_{v}
\end{array}\right)_{r e c}=\frac{e^{-j k R}}{k R}\left(\begin{array}{cc}
S_{h h} & S_{h v} \\
S_{v h} & S_{v v}
\end{array}\right)\left(\begin{array}{c}
E_{h} \\
E_{v}
\end{array}\right)_{\text {ill }}
$$

where $S_{h h}, S_{h v}$, and $S_{v v}$ denotes sending and receiving polarizations of scattering components while $E_{h}, E_{v}$ denotes EM signals in horizontal and vertical polarizations. $\mathrm{R}$ and $\mathrm{k}$ denotes range between sending antenna to the ground and the wave number of EM signals.

Stokes vector, $\mathbf{J}$ is defined in equation (2).

$J=\left(\begin{array}{c}J_{0} \\ J_{1} \\ J_{2} \\ J_{3}\end{array}\right)=\left(\begin{array}{c}\left|E_{h}\right|^{2}+\left|E_{v}\right|^{2} \\ \left|E_{h}\right|^{2}-\left|E_{v}\right|^{2} \\ 2\left(\operatorname{Re}\left(E_{h} E_{v}^{*}\right)\right) \\ -2\left(\operatorname{Im}\left(E_{h} E_{v}^{*}\right)\right)\end{array}\right)=\left(\begin{array}{c}J_{0} \\ J_{0} \cos (2 \chi) \cos (2 \phi) \\ J_{0} \cos (2 \chi) \sin (2 \phi) \\ J_{0} \sin (2 \chi)\end{array}\right)$

Also Muller matrix is defined in equation (3).

$$
\begin{gathered}
M=R W R^{-1} \\
W=\left(\begin{array}{cccc}
S_{h h} S_{h h}^{*} & S_{h h} S_{h v}^{*} & S_{h v} S_{h h}^{*} & S_{h v} S_{h v}^{*} \\
S_{h h} S_{v h}^{*} & S_{h h} S_{v v}^{*} & S_{h v} S_{v h}^{*} & S_{h v} S_{h v}^{*} \\
S_{v h} S_{h h}^{*} & S_{v h} S_{h v}^{*} & S_{v v} S_{h h}^{*} & S_{v v} S_{h v}^{*} \\
S_{v h} S_{v h}^{*} & S_{v h} S_{v v}^{*} & S_{v v} S_{v h}^{*} & S_{v v} S_{v h}^{*}
\end{array}\right) \\
R=\left(\begin{array}{cccc}
1 & 0 & 0 & 1 \\
1 & 0 & 0 & -1 \\
0 & 1 & 1 & 0 \\
0 & j & -j & 0
\end{array}\right) \quad U_{4}=\left(\begin{array}{cccc}
1 & 0 & 0 & 0 \\
0 & 1 & 0 & 0 \\
0 & 0 & 1 & 0 \\
0 & 0 & 0 & -1
\end{array}\right) \\
2 U_{4} M_{s}=M \quad M_{s}=\frac{1}{2} U_{4}^{-1} M
\end{gathered}
$$

Elements of the Muller matrix can be calculated with the equation (4),

$$
\begin{aligned}
& M_{11}=\frac{1}{4}\left(S_{h h} S_{h h}^{*}+S_{v v} S_{v v}^{*}+S_{h v} S_{h v}^{*}+S_{v h} S_{v h}^{*}\right) \\
& M_{12}=\frac{1}{4}\left(S_{h h} S_{h h}^{*}-S_{v v} S_{v v}^{*}-S_{h v} S_{h v}^{*}+S_{v h} S_{v h}^{*}\right) \\
& M_{13}=\frac{1}{2} \operatorname{Re}\left(S_{h h} S_{h v}^{*}+S_{v v} S_{v h}^{*}\right) \\
& M_{14}=\frac{1}{2} \operatorname{Im}\left(S_{h h} S_{h v}^{*}+S_{v v} S_{v h}^{*}\right) \\
& M_{21}=\frac{1}{4}\left(S_{h h} S_{h h}^{*}-S_{v v} S_{v v}^{*}+S_{h v} S_{h v}^{*}-S_{v h} S_{v h}^{*}\right) \\
& M_{22}=\frac{1}{4}\left(S_{h h} S_{h h}^{*}+S_{v v} S_{v v}^{*}-S_{h v} S_{h v}^{*}-S_{v h} S_{v h}^{*}\right) \\
& M_{23}=\frac{1}{2} \operatorname{Re}\left(S_{h h} S_{h v}^{*}-S_{v h} S_{v v}^{*}\right) \\
& M_{24}=\frac{1}{2} \operatorname{Im}\left(S_{h h} S_{h v}^{*}+S_{v h} S_{v v}^{*}\right) \\
& M_{31}=\frac{1}{2} \operatorname{Re}\left(S_{h h} S_{v h}^{*}+S_{h v} S_{v v}^{*}\right) \\
& M_{32}=\frac{1}{2} \operatorname{Re}\left(S_{h h} S_{v h}^{*}-S_{h v} S_{v v}^{*}\right) \\
& M_{33}=\frac{1}{2} \operatorname{Re}\left(S_{h h} S_{v v}^{*}\right)+\frac{1}{4}\left(S_{v h} S_{h v}^{*}+S_{h v} S_{v h}^{*}\right) \\
& M_{34}=\frac{1}{2} \operatorname{Im}\left(S_{h h} S_{v v}^{*}+S_{h v} S_{v h}^{*}\right) \\
& M_{41}=\frac{1}{2} \operatorname{Im}\left(S_{h h} S_{v h}^{*}+S_{h v} S_{v v}^{*}\right) \\
& M_{42}=\frac{1}{2} \operatorname{Im}\left(S_{h h} S_{v h}^{*}-S_{h v} S_{v v}^{*}\right) \\
& M_{43}=\frac{1}{2} \operatorname{Im}\left(S_{h h} S_{v v}^{*}-S_{h v} S_{v h}^{*}\right) \\
& M_{44}=-\frac{1}{2} \operatorname{Re}\left(S_{h h} S_{v v}^{*}\right)+\frac{1}{4}\left(S_{v h} S_{h v}^{*}+S_{h v} S_{v h}^{*}\right)
\end{aligned}
$$

Meanwhile polarization signature can be defined as follows,

$$
\sigma_{0}=C J_{r}^{T}<M_{s}>J_{t}=\sigma_{0}\left(\chi_{r}, \psi_{r}, \chi_{t}, \psi_{t}\right) \quad C: \text { const. }
$$

Thus polarization signature can be characterized with $\chi, \psi$ as shown in Figure 1.

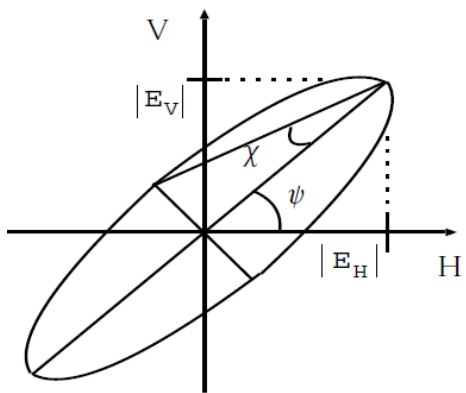

Fig.1. Characterization of polarization signature

\section{B. Full Polarimetric SAR Imagery Data Decompositions}

Full polarimetric SAR is defined as the SAR which allows acquisition the scattering components of which polarimetric SAR sends Electric Magnetic: EM signals to the ground cover targets in horizontal and vertical polarizations and also that receives EM signals from the ground cover targets in horizontal and vertical polarizations. Therefore, scattering matrix of equation (5) can be obtained. 
$X=\left[S_{h h} S_{h v} S_{v v}\right]^{T}$

Where $S_{i j}$ denotes scattering coefficients while the first and the second suffix of $S_{h h}, S_{h v}$, and $S_{v v}$ denotes sending and receiving polarizations.

$$
\begin{aligned}
& C=\left\langle X X^{* T}\right\rangle \\
& C=\lambda_{1} K_{1}\left(K_{1}\right)^{*^{T}}+\lambda_{2} K_{2}\left(K_{2}\right)^{*^{T}}+\lambda_{3} K_{3}\left(K_{3}\right)^{*^{T}}
\end{aligned}
$$

$K_{l}, K_{2}, K_{3}$ denotes Single scattering coefficient (Odd), Double scattering coefficient (Even), and Defuse scattering coefficient (Defuse) while $\lambda_{1}, \lambda_{2}, \lambda_{3}$ denotes eigen values for $K_{l}$, $K_{2}, K_{3}$. Ratio of these three variables can be determined by the following contribution factors

$$
\lambda i /\left(\lambda_{1}+\lambda_{2}+\lambda_{3}\right),(i=1,2,3)
$$

Not only these three features but also the other features can be decomposed. Circular polarization of EM wave, $S_{L R}, S_{L L}$, $S_{R R}, S_{R L}$ can be derived from $S_{i j}$ as follows,

$$
\begin{aligned}
& S_{R R}=\frac{1}{2}\left(S_{h h}-S_{v v}+j 2 S_{h v}\right) \\
& S_{L L}=\frac{1}{2}\left(S_{h h}-S_{v v}-j 2 S_{h v}\right) \\
& S_{L R}=\frac{1}{2}\left(S_{h h}+S_{v v}\right)
\end{aligned}
$$

Linear and circular polarization is illustrated in Figure 2.

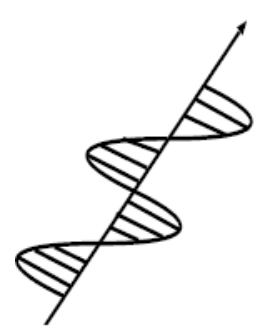

(a)Horizontal Polarization

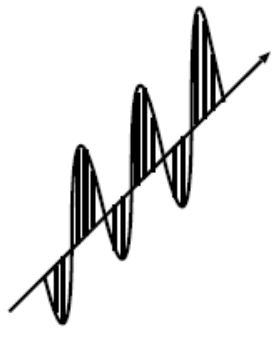

(b) Vertical Polarization (c)Circular

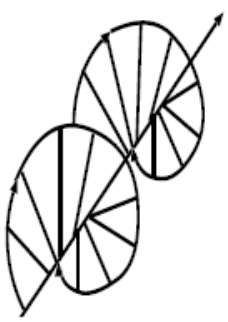
Polarization
Fig.2. Polarization features

Then Sphere, Di-plane and Helix components are defined as follows,

$K_{s}=\left|S_{L R}\right| \quad K_{d}=\left|S_{L L}\right| \quad K_{h}=\left|S_{R R}\right|-\left|S_{L L}\right|$ for $\left|S_{R R}\right|>\left|S_{L L}\right|$ $K_{s}=\left|S_{L R}\right| \quad K_{d}=\left|S_{R R}\right| \quad K_{h}=\left|S_{R R}\right|-\left|S_{L L}\right|$ for $\left|S_{R R}\right|<\left|S_{L L}\right|$

Contribution factors for each component can be calculated as follows,

$K_{i} /\left(K_{s}+K_{d}+K_{h}\right),(i=s, d, h)$

Namely, this decomposition can be done with eigen value decomposition of covariance matrix of scattering matrix.

Other than these, there is another decomposition method which allows extract odd number scattering component, even number scattering component, Brag scattering component, orthogonal polarization scattering component, etc. can be extracted from $S_{i j .}$ Furthermore, there are some other decomposition methods for not only covariance matrix but also coherence matrix as well.

\section{Classification Method Used}

There are many image classification methods with features. Probability Density Function: PDF of the extracted features are followed by normal distribution, usually. Therefore, widely used Maximum Likelihood Classification: MLH method assuming normal distribution is used.

\section{Correlation Matrix}

Through correlation analysis, correlation coefficients among the extracted features are calculated and create correlation matrix. Then summation of correlation matrix elements is calculated. This variable is referred to SUM hereafter. After that, sorting of the summation of correlation matrix elements is performed. Small summation of correlation matrix elements implies independent feature or effective feature for classification.

\section{E. Overlapped Portion of Probability Density Function between Features}

Another measure for effectiveness features, or features can be assessed with overlapped portion of PDFs between two features. If the overlapped portion is small, then it is effective to image classification.

\section{EXPERIMENTS}

\section{A. Polarimetric SAR Image Data Used}

The PI-SAR (Polarimetric and Interferometric SAR) data of Tsukuba in Japan which was acquired by CRL (Communication Research Laboratory, current NICT: National Institute of Communication Technology) and NASDA (National Space Development Agency of Japan, current JAXA: Japan Aeronautics Exploration Agency) on 23 February 1999 was used. The major characteristics of the PISAR are in Table 1.

TABLE I. MAJOR CHARACTERISTICS OF PI-SAR

\begin{tabular}{|l|l|}
\hline Instrument & NASDA/L-band SAR \\
\hline Center frequency & $1.27 \mathrm{GHz}$ \\
\hline Peak power & $3.5 \mathrm{~kW}$ \\
\hline Band width & $50 \mathrm{MHz}$ \\
\hline Antenna size & $1.6 \mathrm{~m} \times 0.7 \mathrm{~m}$ \\
\hline Polarization & $\mathrm{HH} / \mathrm{HV} / \mathrm{VH} / \mathrm{VV}($ Full Pol. $)$ \\
\hline Incident angle & $20-60 \mathrm{degrees}($ Fixed) \\
\hline Swath width & $42.5 \mathrm{~km}$ \\
\hline Spatial resolution & $3 \mathrm{~m}$ \\
\hline Quantization bit & $8 \mathrm{bits}(\mathrm{I}$ and Q) \\
\hline
\end{tabular}

From the data of SSC (Single-look Slant-range Complex) of the data, the imagery data of PISAR used for experiments is created. Figure 3 shows the imagery data used for the experiments of Okhotsk Sea. 
Intensive Study Area is situated in the Sea of Okhotsk. The data is acquired on 23 February 1999. The imagery data is created with Single Look Slant Range of Complex Data. The data is reprocessed with 8 look processing and is composed with $1000 \times 987$ pixels. It is acquired with PISAR instrument with the parameters of $\mathrm{L}$ band of $\mathrm{HH}$ polarization which is onboard the aircraft. In Figure 3, Green colored areas show Open Water while Black colored areas show Rough Surface Ice. On the other hand, Blue colored areas show Thin Ice while White colored areas show Smooth Surface Ice.

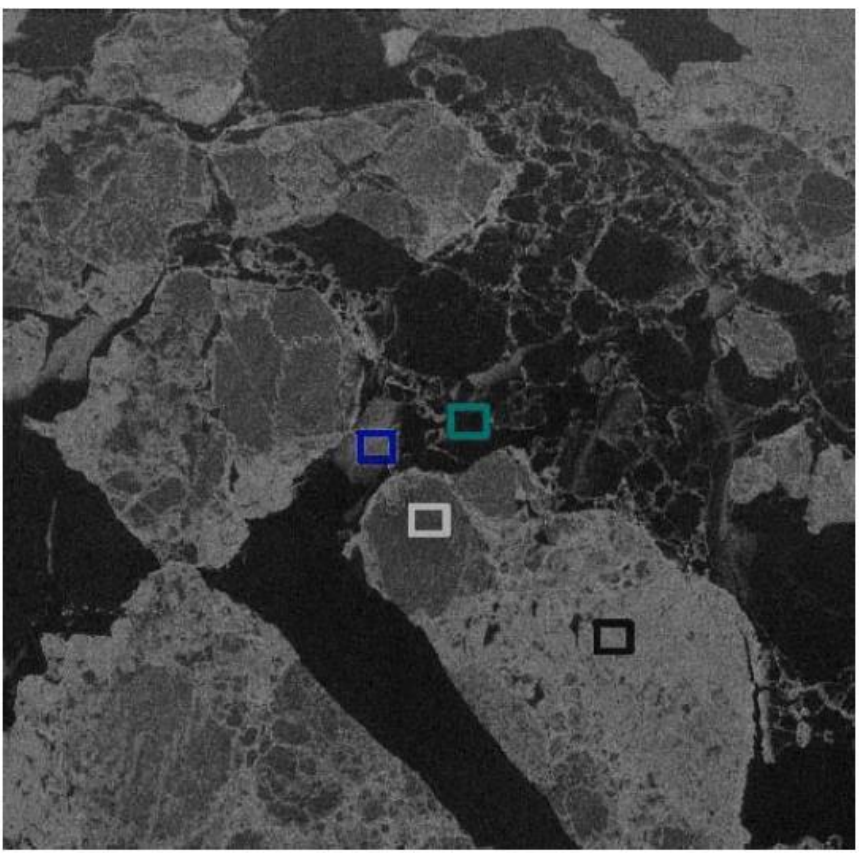

Fig.3. Intensive Study Area in the sea of Okhotsk 23 Feb.1999(Single look Slant range Complex Data,8look $1000 \times 987$ pixels,L band HH polarization)

Green: Open water, Black: Rough surface ice, Blue Thin ice White: Smooth surface ice.

\section{B. Correlation Matrix}

Correlation matrix for open water is shown in Table 2 while that of rough first year ice is shown in Table 3, respectively.. Meanwhile, correlation matrix for smooth first year ice is shown in Table 4 while that for thin ice is shown in Table 5, respectively. Two rows from the bottom shows SUM and $|\mathrm{SUM}|$, respectively.

It is found that the smallest SUM for open water is Sphere followed by Diffuse and Helix while that for rough surface of first year ice is Odd number of scattering followed by Even number of scattering and Sphere. It is also found that the smallest SUM for smooth surface of first year ice is Sphere followed by Odd number scattering and Helix as well as Diffuse while that of thin ice is Odd number of scattering followed by Sphere and Even number of scattering. It totally depends on the scattering mechanism for the ground cover targets.

TABLE II. CORRELATION MATRIX FOR OPEN WATER

\begin{tabular}{|c|r|r|r|r|r|r|r|r|c|}
\hline open water & \multicolumn{1}{|c|}{ Odd } & Even & Diffuse & Sphere & Diplane & Helix & HH & HV & VV \\
\hline Od & 1 & -0.99 & -0.49 & 0.399 & -0.22 & -0.3 & 0.527 & 0.13 & 0.608 \\
\hline Ev & -0.99 & 1 & 0.402 & -0.37 & 0.233 & 0.233 & -0.49 & -0.18 & -0.57 \\
\hline Df & -0.49 & 0.402 & 1 & -0.41 & -0.05 & 0.66 & -0.56 & 0.39 & -0.58 \\
\hline Sp & 0.399 & -0.37 & -0.41 & 1 & -0.74 & -0.48 & 0.504 & -0.06 & 0.377 \\
\hline Dp & -0.22 & 0.233 & -0.05 & -0.74 & 1 & -0.23 & -0.22 & -0.19 & -0.09 \\
\hline Hx & -0.3 & 0.233 & 0.66 & -0.48 & -0.23 & 1 & -0.44 & 0.32 & -0.43 \\
\hline HH & 0.527 & -0.49 & -0.56 & 0.504 & -0.22 & -0.44 & 1 & 0.38 & 0.93 \\
\hline HV & 0.13 & -0.18 & 0.385 & -0.06 & -0.19 & 0.322 & 0.38 & 1 & 0.403 \\
\hline VV & 0.608 & -0.57 & -0.58 & 0.377 & -0.09 & -0.43 & 0.93 & 0.4 & 1 \\
\hline & 0.07 & -0.1 & 0.04 & 0.02 & -0.1 & 0.04 & 0.18 & 0.24 & 0.18 \\
& 0.07 & 0.08 & 0.04 & 0.02 & 0.06 & 0.04 & 0.18 & 0.24 & 0.18 \\
\hline
\end{tabular}

TABLE III. CORRELATION MATRIX FOR ROUGH FIRST YEAR ICE

\begin{tabular}{|c|r|r|r|r|r|r|r|r|c|}
\hline rough FYI & Odd & Even & Diffuse & Sphere & Diplane & Helix & HH & HV & VV \\
\hline Od & 1 & -0.99 & -0.32 & 0.621 & -0.51 & -0.29 & 0.286 & -0.04 & 0.349 \\
\hline Ev & -0.99 & 1 & 0.174 & -0.56 & 0.502 & 0.215 & -0.26 & -0.05 & -0.32 \\
\hline Df & -0.32 & 0.174 & 1 & -0.53 & 0.193 & 0.545 & -0.2 & 0.59 & -0.29 \\
\hline Sp & 0.621 & -0.56 & -0.53 & 1 & -0.74 & -0.57 & 0.332 & -0.24 & 0.402 \\
\hline Dp & -0.51 & 0.502 & 0.193 & -0.74 & 1 & -0.13 & -0.3 & -0.07 & -0.32 \\
\hline Hx & -0.29 & -0.215 & 0.545 & -0.57 & -0.13 & 1 & -0.13 & 0.44 & -0.2 \\
\hline HH & \multicolumn{1}{|c|}{0.286} & -0.26 & -0.2 & 0.332 & -0.3 & -0.13 & 1 & 0.52 & 0.789 \\
\hline HV & -0.04 & -0.05 & 0.586 & -0.24 & -0.07 & 0.437 & 0.519 & 1 & 0.44 \\
\hline VV & 0.349 & -0.32 & -0.29 & 0.402 & -0.32 & -0.2 & 0.789 & 0.44 & 1 \\
\hline & 0.01 & -0 & 0.13 & -0 & -0 & 0.1 & 0.23 & 0.29 & 0.21 \\
& 0.01 & 0.03 & 0.13 & 0.03 & 0.04 & 0.1 & 0.23 & 0.29 & 0.21 \\
\hline
\end{tabular}

TABLE IV. CORRELATION MATRIX FOR SMOOTH FIRST YEAR ICE

\begin{tabular}{|c|r|r|r|r|r|r|r|r|c|}
\hline smooth FYI & Odd & Even & Diffuse & Sphere & Diplane & Helix & HH & HV & VV \\
\hline Od & 1 & -0.99 & -0.55 & 0.693 & -0.53 & -0.35 & 0.537 & -0.04 & 0.629 \\
\hline Ev & -0.99 & 1 & 0.414 & -0.65 & 0.546 & 0.271 & -0.48 & -0.06 & -0.58 \\
\hline Df & -0.55 & 0.414 & 1 & -0.55 & 0.179 & 0.589 & -0.57 & 0.53 & -0.59 \\
\hline Sp & 0.693 & -0.65 & -0.55 & 1 & -0.75 & -0.53 & 0.638 & -0.14 & 0.597 \\
\hline Dp & -0.53 & 0.546 & 0.179 & -0.75 & 1 & -0.16 & -0.43 & -0.18 & -0.37 \\
\hline Hx & -0.35 & 0.271 & 0.589 & -0.53 & -0.16 & 1 & -0.39 & 0.43 & -0.42 \\
\hline HH & 0.537 & -0.48 & -0.57 & 0.638 & -0.43 & -0.39 & 1 & 0.15 & 0.834 \\
\hline HV & -0.04 & -0.06 & 0.535 & -0.14 & -0.18 & 0.433 & 0.146 & 1 & 0.14 \\
\hline VV & 0.629 & -0.58 & -0.59 & 0.597 & -0.37 & -0.42 & 0.834 & 0.14 & 1 \\
\hline & 0.04 & -0.1 & 0.05 & 0.03 & -0.1 & 0.05 & 0.14 & 0.2 & 0.14 \\
& 0.04 & 0.06 & 0.05 & 0.03 & 0.08 & 0.05 & 0.14 & 0.2 & 0.14 \\
\hline
\end{tabular}


TABLE V. CORRELATION MATRIX FOR THIN ICE

\begin{tabular}{|c|r|r|r|r|r|r|r|r|c|}
\hline thin ice & Odd & Even & Diffuse & Sphere & Diplane & Helix & HH & HV & VV \\
\hline Od & 1 & -0.99 & -0.34 & 0.656 & -0.48 & -0.31 & 0.331 & -0.15 & 0.44 \\
\hline Ev & -0.99 & 1 & 0.193 & -0.61 & 0.493 & 0.235 & -0.28 & 0.04 & -0.38 \\
\hline Df & -0.34 & 0.193 & 1 & -0.47 & 0.074 & 0.533 & -0.42 & 0.73 & -0.51 \\
\hline Sp & 0.656 & -0.61 & -0.47 & 1 & -0.68 & -0.54 & 0.533 & -0.27 & 0.537 \\
\hline Dp & -0.48 & 0.493 & 0.074 & -0.68 & 1 & -0.26 & -0.28 & -0.13 & -0.25 \\
\hline Hx & -0.31 & 0.235 & 0.533 & -0.54 & -0.26 & 1 & -0.38 & 0.51 & -0.42 \\
\hline HH & 0.331 & -0.28 & -0.42 & 0.533 & -0.28 & -0.38 & 1 & -0.06 & 0.425 \\
\hline HV & -0.15 & 0.044 & 0.73 & -0.27 & -0.13 & 0.513 & -0.06 & 1 & -0.11 \\
\hline VV & 0.44 & -0.38 & -0.51 & 0.537 & -0.25 & -0.42 & 0.425 & -0.11 & 1 \\
\hline & 0.02 & -0 & 0.09 & 0.02 & -0.1 & 0.04 & 0.1 & 0.17 & 0.08 \\
& 0.02 & 0.03 & 0.09 & 0.02 & 0.06 & 0.04 & 0.1 & 0.17 & 0.08 \\
\hline
\end{tabular}

\section{Overlapped Portion of Probability Density Function between Features}

Figure 4 shows PDF of each class for each feature extracted from the full polarization SAR and decomposed components from the full polarization of SAR. Some of the features, in particular, odd and even number of scattering as well as diffuse component shows not normal distribution at all. Therefore, it would better to check not only correlation coefficients but also PDF of the features.

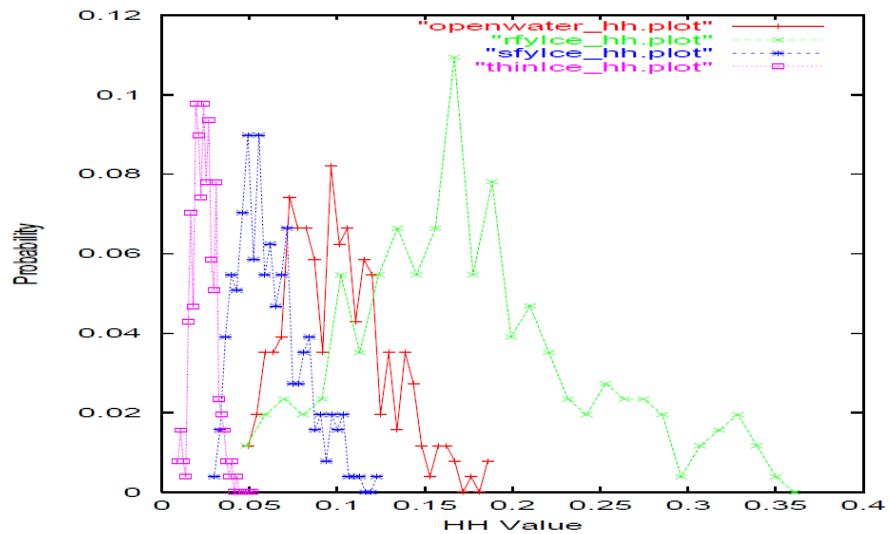

(a) $\mathrm{HH}$

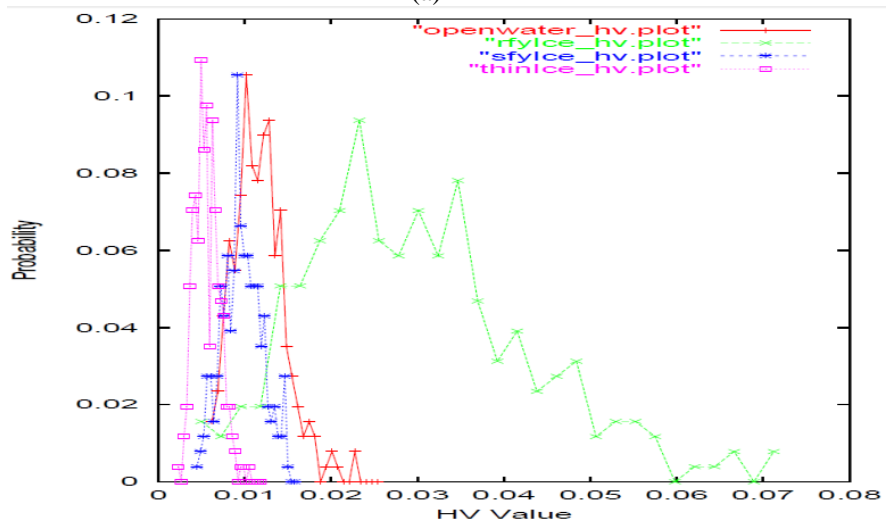

(b) $\mathrm{HV}$

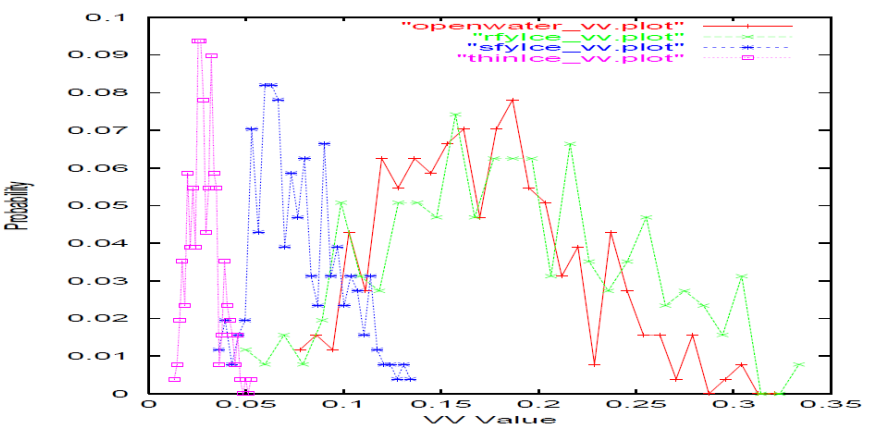

(c) $\mathrm{VV}$

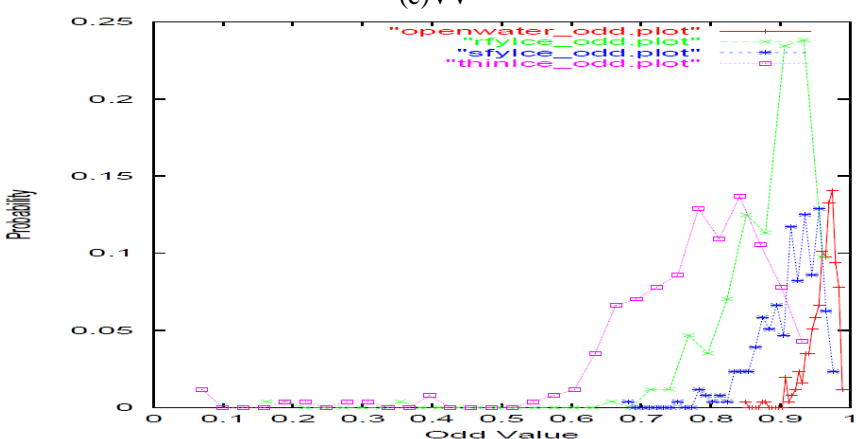

(d)Odd number scattering

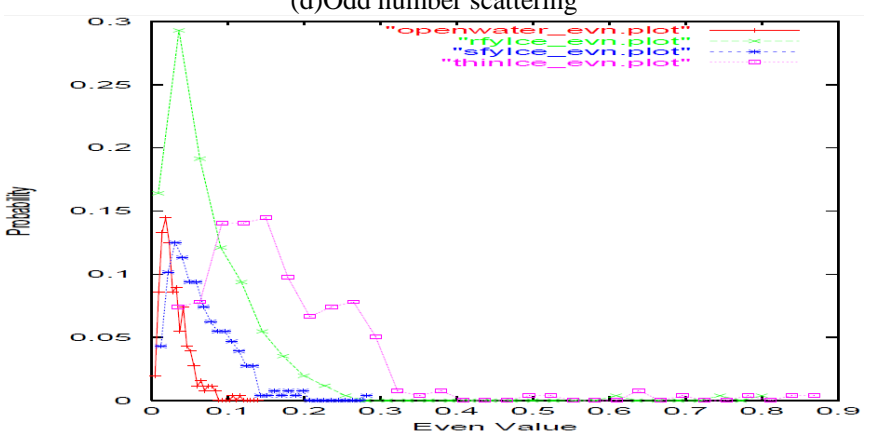

(e)Even number of scattering
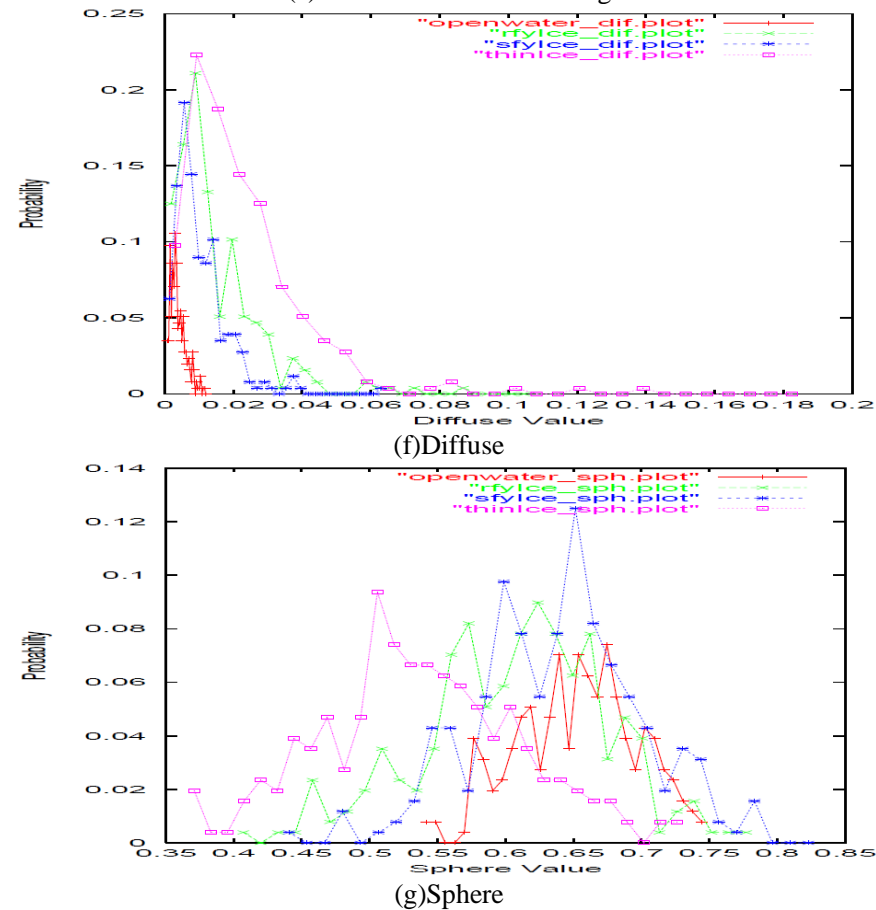


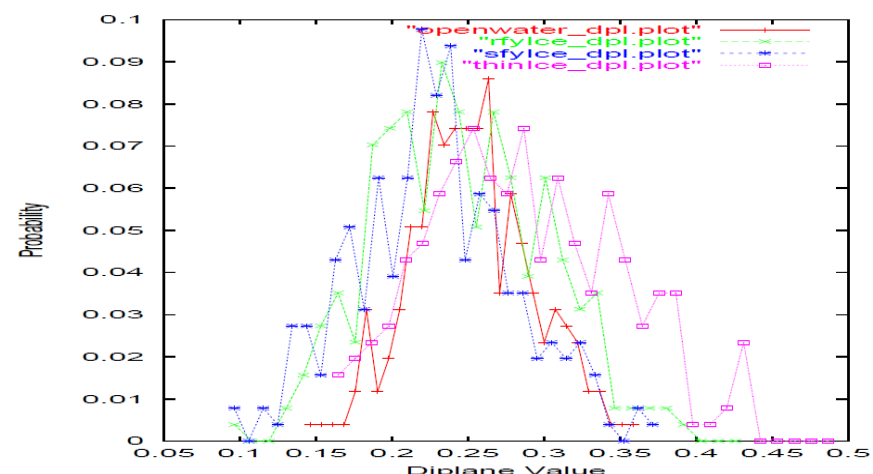

(h)Di-plane

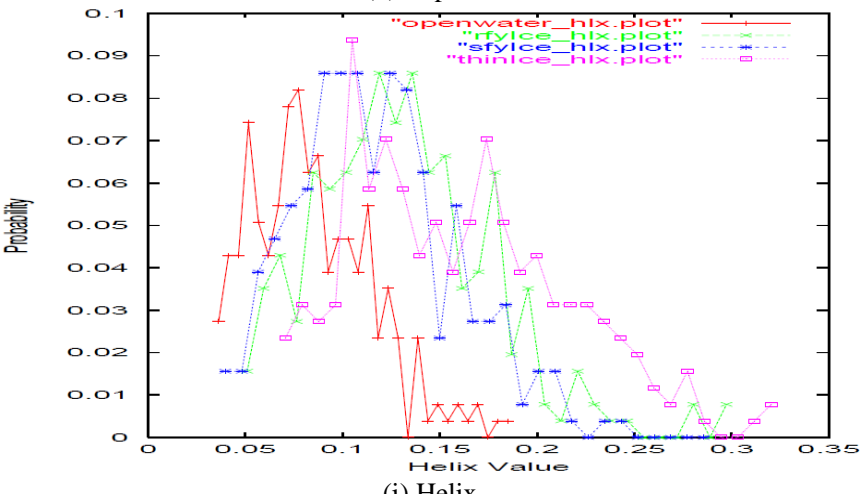

(i) Helix

Fig.4. PDF of each class for each feature

\section{Confusion Matrix}

Nevertheless correlation among the received power signals of $\mathrm{HH}, \mathrm{HV}$, and $\mathrm{VV}$, classification result shows highest Percent Correct Classification: PCC of $94.6 \%$ among the all possible combination of receiving power signals and the decomposed components. Figure 5 shows the classified resultant image.

TABLE VI. CONFUSION MATRIX FOR UTILIZING THREE RECEIVED POSERS ONLY $(\mathrm{PCC}=94.6 \%)$

\begin{tabular}{|l|c|c|c|c|}
\hline & OpenWater & RoughIce & SmoothIce & ThinIce \\
\hline OpenWater & 92.2 & 0.8 & 7.0 & 0.0 \\
\hline RoughIce & 2.0 & 92.2 & 5.8 & 0.0 \\
\hline SmoothIce & 3.1 & 0.4 & 95.3 & 1.2 \\
\hline ThinIce & 0.0 & 0.0 & 1.2 & 98.8 \\
\hline
\end{tabular}

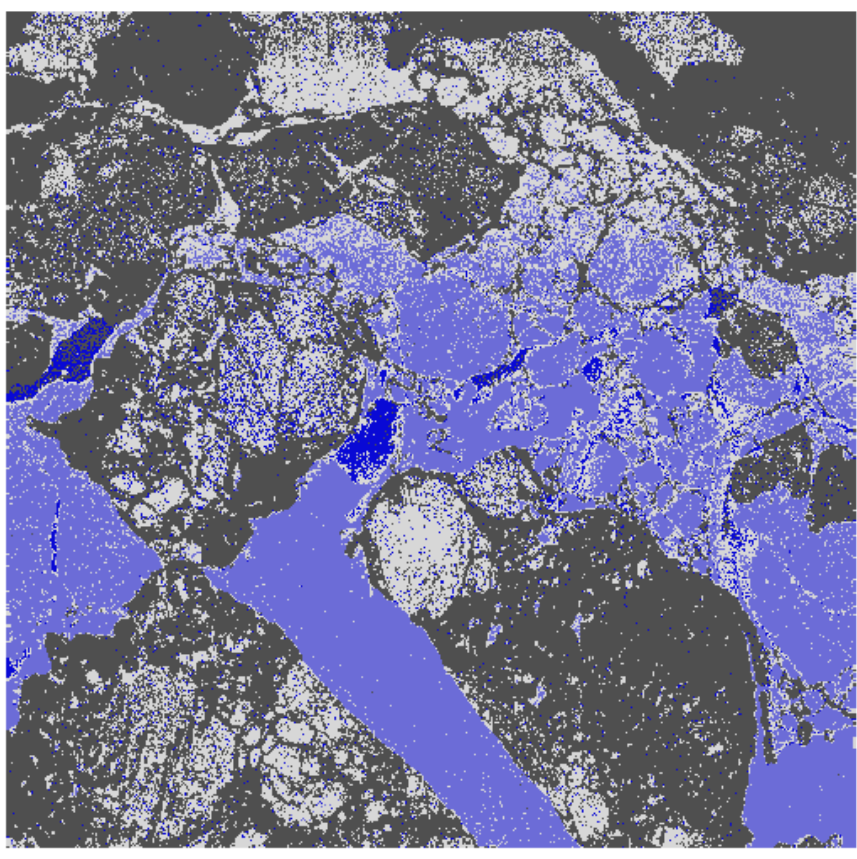

Fig.5. Classification resultant image for utilizing three received power singles only (Black: Rough surface ice, White: Smooth surface ice, Light blue: Open water, Dark blue: Thin ice)

The next highest PCC is achieved by the combination of three receiving power signals and Diffuse as well as Odd number of scattering components. Figure 6 shows classified resultant image.

TABLE VII. CONFUSION MATRIX FOR UTILIZING THREE RECEIVED POWERS AND ODD NUMBER OF SCATTERING AS WELL AS DIFFUSE COMPONENT (PCC $=93.6 \%$ )

\begin{tabular}{|l|c|c|c|c|}
\hline & OpenWater & RoughIce & SmoothIce & ThinIce \\
\hline OpenWater & 93.4 & 0.8 & 5.8 & 0.0 \\
\hline RoughIce & 3.1 & 92.2 & 4.7 & 0.0 \\
\hline SmoothIce & 6.6 & 0.8 & 92.2 & 0.4 \\
\hline ThinIce & 0.8 & 0.0 & 3.5 & 95.7 \\
\hline
\end{tabular}

Although PCC of the classification with three received power signals and Diffuse and Odd number of scattering components is lower than that with just three received power signals only, there are some thin ice pixels are observed in the open water and smooth surface of first year ice areas. 


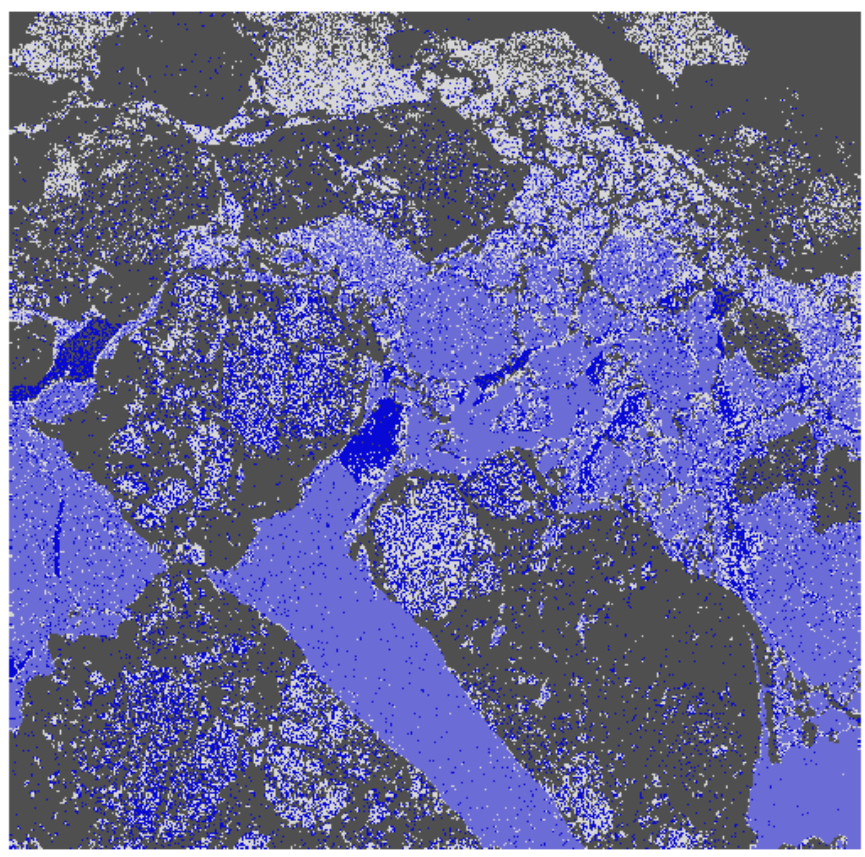

Fig.6. Classification resultant image for utilizing three received power singles and Diffuse, Odd number scattering components. (Black: Rough surface ice, White: Smooth surface ice, Light blue: Open water, Dark blue: Thin ice)

This is natural. Therefore, classified results from the classification with three received power signals and Diffuse and Odd number of scattering components shows much reliable classified results.

As the results, it is found that three receiving power signals plus Diffuse and Odd number of scattering component is effective to the sea ice classifications. The reasons for this are

1) surface scattering is dominant for the smooth and rough surface first year ice.

2) Diffuse component of smooth surface first year ice is smaller than that of rough surface first year ice.

On the other hand, thin ice consists of a kind of layered material with open water which is covered with ice so that it is hard to discriminate thin ice from smooth surface first year ice as well as open water.

\section{CONCLUSION}

Multi spectral image classification method with selection processes of independent spectral features through correlation analysis is proposed. The proposed method is validated by applying to the polarimetric Synthetic Aperture Radar: SAR data. Also Probability Distribution Function: PDF for of features are checked and confirmed the most independent PDF allows greatest classification performance.

It is found that three receiving power signals plus Diffuse and Odd number of scattering component is effective to the sea ice classifications. The reasons for this are (1) surface scattering is dominant for the smooth and rough surface first year ice. (2)Diffuse component of smooth surface first year ice is smaller than that of rough surface first year ice.

On the other hand, thin ice consists of a kind of layered material with open water which is covered with ice so that it is hard to discriminate thin ice from smooth surface first year ice as well as open water.

\section{ACKNOWLEDGMENT}

The author would like to thank Mr. Saeki for his efforts through experiments and simulations.

\section{REFERENCES}

[1] Zebker, H.A., vanZyl, J.J. Imaging radar polarimetry: A review. Proc. IEEE 79, 1583-1606, 1991.

[2] Ulaby, F.T., Elachi, C. Radar Polarimetry for Geoscience Applications.Artech House Inc., Dedham, 1990.

[3] Mott, M. Antennas for Radar Communications - A Polarimetric Approach. John Wiley, New York, 1992.

[4] Henderson, F.M., Lewis, A.J. Principles and applications of imaging radar, in: Manual of Remote Sensing, third ed. John Wiley, New York, 1998.

[5] Krogager, E. Analysis of the absolute and relative phase conditions of transforming matrices for the sinclair and covariance matrices in radar polarimetry. J. Opt. Soc. Am. E72B, 1A, 1993.

[6] Dong, Y. Imaging radar polarimeter from wave synthesis. J. Geophys. Res. 92 (81), 638-701, 1987.

[7] Zebker, H.A., et al., Imaging radar polarimeter from wave synthesis, J. Geophysical Research, Vol.92, No.81, pp.638-701, 1987.

[8] Krogager, E., Czyz, Z.H. Properties of the sphere, di-plane, helix decomposition, in: Proceedings, Third International Workshop on Radar Polarimetry, vol. 1, pp. 106-114, 1995.

[9] Cloude, S.R., Pottier, E. A review of target decomposition theorems in radar polarimetry. IEEE Trans. Geosci. Remote Sensing 34 (2), 498-518, 1995.

[10] Pottier, E., Lee, J.S. Unsupervised classification scheme of Polsar images based on the complex Wishart distribution and H/A/a polarimetric decomposition theorems, Proceedings of the Third European Conference on Synthetic Aperture Radar: EUSAR, 2000.

[11] Scheuchl, B., Hajinsek, I., Cumming, I.G., et al. Sea ice classification using multi-frequency polarimetric SAR data, in: Proceedings of the IGARSS'02, 2002.

[12] vanZyl, J.J., Zebker, H.A., Elachi, C. Imaging radar polarization signatures, theory and observation. Radio Sci. 22 (4), 529-543, 1987.

[13] Kohei Arai and J.Wang, Polarimetric SAR image classification with maximum curvature of the trajectory in eigen space domain on the polarization signature, Advances in Space Research, 39, 1, 149-154, 2007.

\section{AUTHORS PROFILE}

Kohei Arai, He received BS, MS and PhD degrees in 1972, 1974 and 1982, respectively. He was with The Institute for Industrial Science and Technology of the University of Tokyo from April 1974 to December 1978 also was with National Space Development Agency of Japan from January, 1979 to March, 1990. During from 1985 to 1987, he was with Canada Centre for Remote Sensing as a Post Doctoral Fellow of National Science and Engineering Research Council of Canada. He moved to Saga University as a Professor in Department of Information Science on April 1990. He was a councilor for the Aeronautics and Space related to the Technology Committee of the Ministry of Science and Technology during from 1998 to 2000 . He was a councilor of Saga University for 2002 and 2003. He also was an executive councilor for the Remote Sensing Society of Japan for 2003 to 2005 . He is an Adjunct Professor of University of Arizona, USA since 1998. He also is Vice Chairman of the Commission "A" of ICSU/COSPAR since 2008. He wrote 30 books and published 322 journal papers 